\title{
ENERGY RESOURCE SCHEDULING CONSIDERING REACTIVE POWER MANAGEMENT
}

\author{
Hugo MORAIS \\ GECAD / ISEP \\ Polytechnic of Porto - Portugal \\ hgvm@isep.ipp.pt
}

\author{
Zita VALE \\ GECAD / ISEP \\ Polytechnic of Porto - Portugal \\ zav@isep.ipp.pt
}

\author{
Tiago Sousa \\ GECAD / ISEP \\ Polytechnic of Porto - Portugal \\ tas@isep.ipp.pt
}

\begin{abstract}
The reactive power management is an important task in future power systems. The control of reactive power allows the increase of distributed energy resources penetration as well as the optimal operation of distribution networks. Currently, the control of reactive power is only controlled in large power units and in high and very high voltage substations. In this paper a reactive power control in smart grids paradigm is proposed, considering the management of distributed energy resources and of the distribution network by an aggregator namely Virtual Power Player (VPP).
\end{abstract}

\section{INTRODUCTION}

The increasing use of distributed generation units and other distributed energy resources implies several changes in distribution network operation methodologies. Bidirectional power flows and the uncertainty in bus voltage profiles bring new challenges to system operators [1]. The control of active and reactive power is essential to maintain the operation within the established technical limits. Traditionally, reactive power is not remunerated by the system operator, but a minimum reactive generation is imposed for each unit in peakhours [2]. The main goal is to reduce the branches power flow and therefore the global power losses.

With a correct control of active and reactive power it is possible to increase distributed energy resources penetration [3-4]. An aggregator player called Virtual Power Player (VPP) is proposed to manage distinct energy resources. VPP can manage all types of distributed energy resources, including distributed generation, demand response and storage units [5]. Presently, in the medium and low voltage levels, the distributed energy resources only supply active power. However, in the future, each resource can contribute to the network stability, balancing the active and reactive power supply.

This paper present an energy resources management algorithm that aims at maximizing the VPP profit and that considers the four different reactive power management options in the scheduling process. The main goal is to operate a distribution network with high penetration of distributed generation, demand response and energy storage systems. The network constraints are also considered, namely the line thermal limits and the bus voltage magnitude limits. The consumers and storage participation are considered with different values according to the consumers' size and profile. The envisaged problem is a mixed-integer non-linear problem and has been implemented in Matrix Laboratory (MATLAB) and General Algebraic Modeling System (GAMS). MATLAB was used as an interface with the user and GAMS was used to solve the optimization problem.

Energy resources management problem scheduling is studied in a 33-bus distribution test system, which has high penetration of distributed generation and storage units, considering different reactive power management techniques.

After the introduction, the second section addresses some important aspects concerning distributed energy resources management and the third section includes a case study with four different reactive power injection control scenarios. Finally, some conclusions are presented in the last section

\section{ENERGY RESOURCES MANAGEMENT IN SMART GRID}

To manage the distributed resources, an energy resources management simulation tool called ERMaS was developed by the authors of this paper. In the scope of ERMaS it is assumed that a VPP is able to operate different distributed energy resources in a specific network area [6]. The VPP must respect the contracts established with the available distributed resources in order to achieve a good energy resource management solution. The ERMaS tool uses historical data and daily forecasted data to support the energy resource management process. These data can be seen in Figure 1, concerning the identification of the different DER that may be available in the network. The data base contains the forecasted values for each hour referring to the day-ahead periods, concerning namely the distributed generation capacity, storage status, available demand response events and network configuration. ERMaS use deterministic and heuristic techniques to solve the energy resources management problem [6-7]. The user can choose the deterministic technique if it is needed an exact result or a heuristic technique (Particle Swarm Optimization, Simulated annealing or Genetic Algorithm) if the user needs results in a short time period [7]. The heuristics techniques have been implemented in the Matrix Laboratory (MATLAB) [8] software, and the deterministic technique has been modeled in the General Algebraic Modeling System (GAMS) [9] software. The MATLAB has also been used as interface with GAMS. In this way, GAMS is only responsible for modeling the deterministic 
technique, and MATLAB organizes the input data and results.

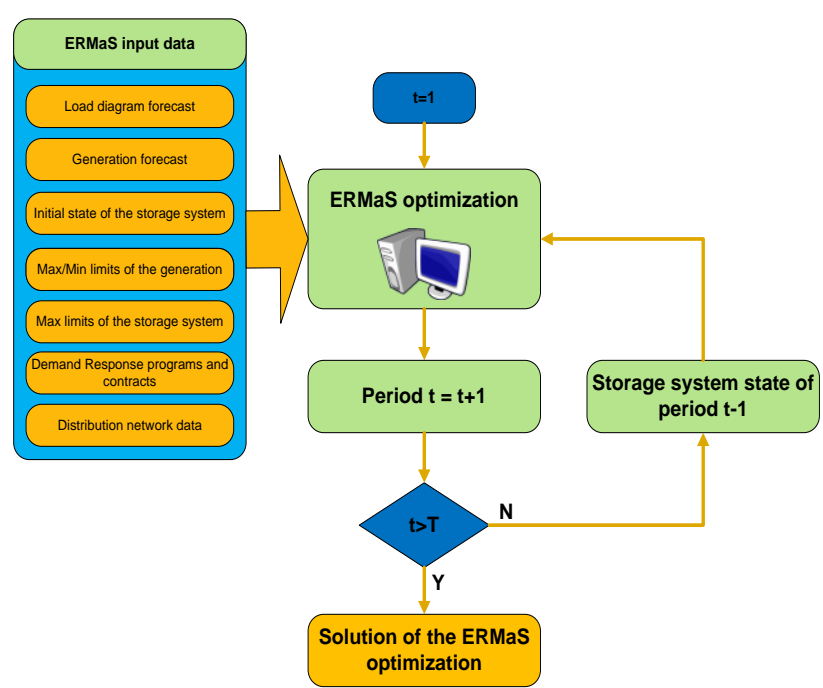

Figure 1. Energy Resources Management flowchart

The main component of the ERMaS tool is the algorithm used to determine the energy resources management. The energy resources management is determined for different periods until it reaches the maximum number of envisaged periods $(T)$. Typically, the energy resource management is executed for the period of 24 hours of the next day [7]. The energy resource management can be performed, in the scope of the ERMaS tool, using deterministic and metaheurisitic techniques [7].

The objective function represents the VPP objectives that present a mathematical significance. The VPP must be able to obtain a minimum cost or a maximum profit through a mathematical representation (objective function) of the involved objectives. In terms of the optimal resource scheduling, the most common approach is developing an objective function that makes possible to maximize its profits.

$$
\begin{aligned}
& \text { Maximize } f= \\
& \qquad \begin{array}{l}
M \sum_{L=1}^{N l} P_{D(L, t)} \times c_{D(L, t)}+\sum_{S=1}^{N_{S}} P_{S t_{-} C(S, t)} \times c_{S t_{-} C(S, t)} \\
\sum_{t=1}^{T} \mid-\sum_{G=1}^{N g} P_{G e n(G, t)} \times c_{G e n(G, t)}-\sum_{S=1}^{N_{S}} P_{S t_{-} D c(S, t)} \times c_{S t_{-} D c(S, t)} \\
\mid-\sum_{L=1}^{N l} P_{D R(L, t)} \times c_{D R(L, t)}
\end{array}
\end{aligned}
$$$$
\forall t \in\{1, \ldots, T\}
$$

Where:

$$
\text { Remuneration from the power demand of load } L
$$
in period $t$

\author{
$\begin{array}{ll}c_{D R(L, t)} & \text { Demand response program cost, for load } L \text { in } \\ \text { period } t\end{array}$

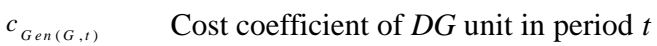 \\ $c_{S t C(S, t)}$ Remuneration from the active power charged in \\ storage $S$ in period $t$ \\ $c_{S t_{-} D c(S, t)} \quad$ Discharge cost of storage $S$ in period $t$ \\ G Generator \\ $L \quad$ Load \\ $\mathrm{Ng} \quad$ Total number of generators \\ $\mathrm{Nl} \quad$ Toatl number of loads \\ Ns Total number of storage \\ $P_{D(L, t)} \quad$ Power demand of load $L$ in period $t$ \\ $P_{G e n(G, t)} \quad$ Active power generation of generation unit $G$ in \\ period $t$ \\ $P_{S t_{-} C(S, t)} \quad$ Active power charge of storage $S$ in period $t$ \\ $P_{S t_{-} D c(S, t)} \quad$ Active power discharge of storage $S$ in period $t$ \\ $P_{D R(L)} \quad$ Active power reduction, for load $L$ at bus $i$ in \\ period $t$ \\ $S \quad$ Storage \\ $t \quad$ Period \\ $T \quad$ Total number of periods
}

However, to validate the effects and the impact of reactive power control, other objective function is proposed, considering the minimization of differences of bus voltage magnitude. The inclusion of demand response variables in objective function is important to avoid the cut of power demand when is not necessary. If the term of demand response is not included, the optimization cut the demand power to reduce the power flow in the lines and, consequently, to reduce the voltage drop which is the main objective of the problem. When the demand response variables is included in the objective function multiplied by an factor $\left(\beta_{L}\right)$ to penalize the use of demand response and the increase of objective function

\begin{tabular}{|c|c|}
\hline$B$ & Bus \\
\hline$N b$ & Total number of bus \\
\hline$V_{(R e f, t)}$ & $\begin{array}{l}\text { Voltage magnitude of system slack bus in period } \\
t\end{array}$ \\
\hline$V_{(B, t)}$ & Voltage magnitude bus $B$ in period $t$ \\
\hline$\beta_{L}$ & $\begin{array}{l}\text { Penalization factor of use of demand response } \\
\text { programs of load } L\end{array}$ \\
\hline
\end{tabular}

$$
\begin{aligned}
& \text { Manimize } f= \\
& \operatorname{Min} \sum_{t=1}^{T}\left\lceil\sum_{B=1}^{N b} V_{(R e f, t)}-V_{(B, t)}+\beta_{L} \times \sum_{L=1}^{N l} P_{D R(L, t)} \times c_{D R(L, t)}\right] \\
& \forall t \in\{1, \ldots, T\}
\end{aligned}
$$

Where: 


\section{CASE STUDY}

The case studies presented in this section use a distribution network with 32 bus that can be found in [10]. Figure 2 shows the evolution of 32 bus distribution network to the year 2040 with, Distributed Generation (DG) spread over the network [10]. In this figure, the solid lines represent the branches that are used, and the dashed lines represent the branches that can be used in a reconfiguration scenario.

In Figure 2 the distributed generation units are represented by different colors, which identify the used generation technology type. Additionally, the installed power is indicated. For the year 2040, this network includes 66 DG units (32 photovoltaic, 15 CHP, 8 fuel cell, 5 wind farm, 3 biomass, 2 small hydro and 1 MSW). For the presented case studies, it is considered that the VPP has the obligation of buying all the generation power from photovoltaic units.

Additionally, two external suppliers which supply electrical energy to this distribution network are considered in the case studies. These external suppliers are connected to bus 0 .

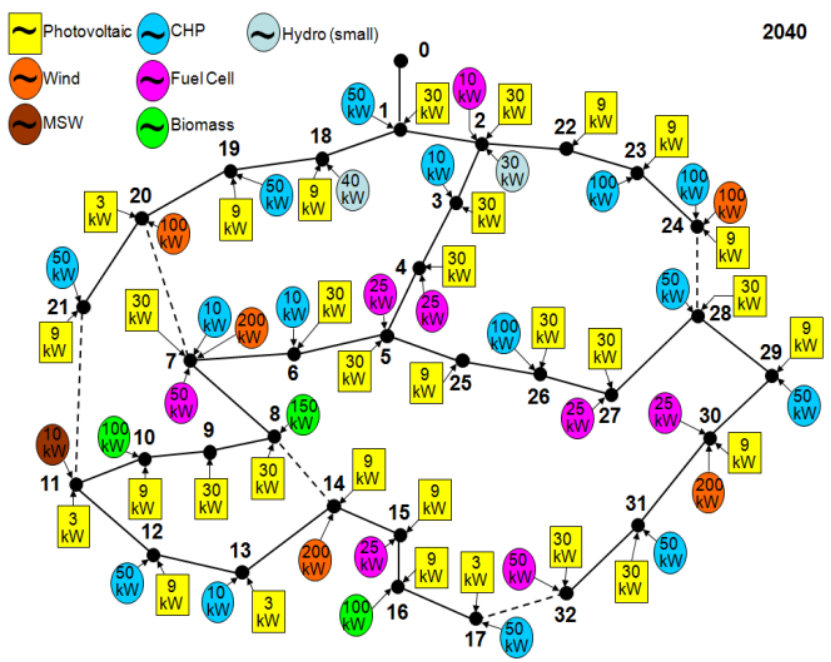

Figure 2. Distribution network configuration in 2040 scenario [10]

Four different scenarios were simulated. In the first one the control of reactive power injected by the distributed generation is not considered. The distributed generation only injects active power in the distribution network, according to the energy resources management scheduling process. In the second scenario is considered the injected of reactive power considering the use of fixed capacitor bank installed in each distributed generation unit. Each unit has capacitor bank with reactive capacity equal to $40 \%$ of active installed capacity. However, these capacitor banks are not controlled by the VPP. This is means that the capacitors banks are always connected and inject reactive power in the distribution network in all scheduling periods.
In the third scenario is considered the control of the reactive power injected in network by the distributed generation, considering the VPP profit maximization objective function. Finally, in the fourth scenario is considered the use of reactive control of distributed generation and the use of minimization of voltage magnitude differences objective function. In all scenarios the bus voltage magnitude limits are $5 \%$. Bus 0 is the reference bus and has a fixed voltage magnitude equal to 1 and the voltage angle equal to 0 .

Table I - Scenarios description

\begin{tabular}{|l|c|c|c|}
\hline \multirow{2}{*}{} & \multirow{2}{*}{$\begin{array}{c}\text { Reactive } \\
\text { Control }\end{array}$} & $\begin{array}{c}\text { Operation } \\
\text { cost }\end{array}$ & $\begin{array}{c}\text { Oboltage } \\
\text { Magnitude } \\
\text { Variance }\end{array}$ \\
\cline { 3 - 4 } & No & Yes & No \\
\hline Scenario 1 & No & Yes & No \\
\hline Scenario 2 & Yes & Yes & No \\
\hline Scenario 3 & Yes & No & Yes \\
\hline Scenario 4 & & & \\
\hline
\end{tabular}

Figure 3 shows the power losses obtained in each simulated scenario.

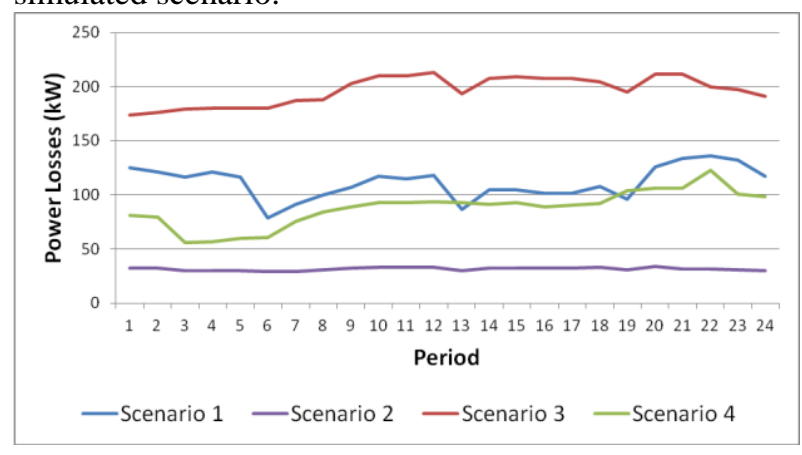

Figure 3. Power Losses in each scenario

Figures 4, 5, 6 and 7 shows the bus voltage magnitude obtained for each simulated scenario. Each period have a different bus voltage magnitude results. However, to improve the perception of the information in the graphics, only the period 1 (off-peak period example) and the period 19 (peak period example) are shown.

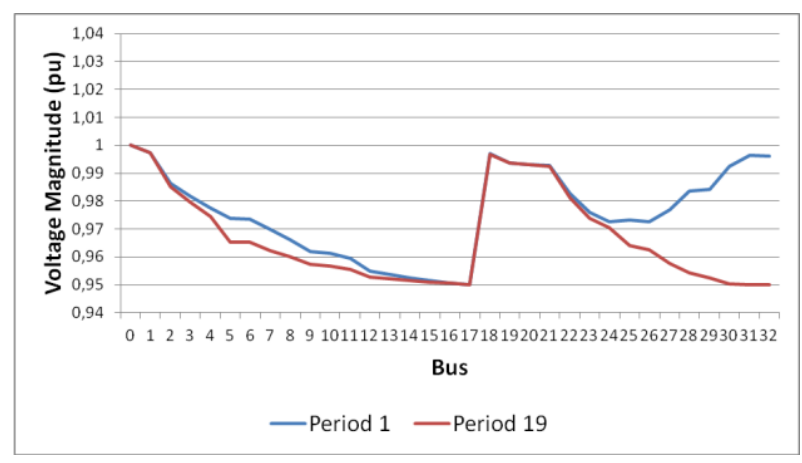

Figure 4. Bus voltage profile in periods 1 and 19 , in scenario 1 


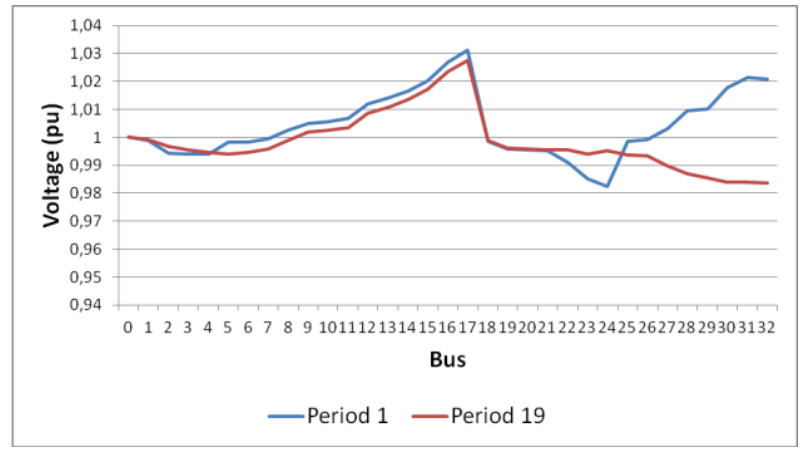

Figure 5. Bus voltage profile in periods 1 and 19 , in scenario 2

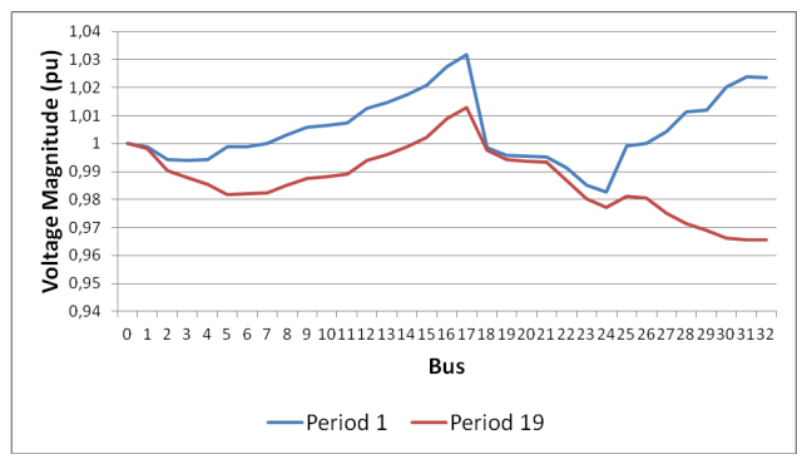

Figure 6. Bus voltage profile in periods 1 and 19, in scenario 3

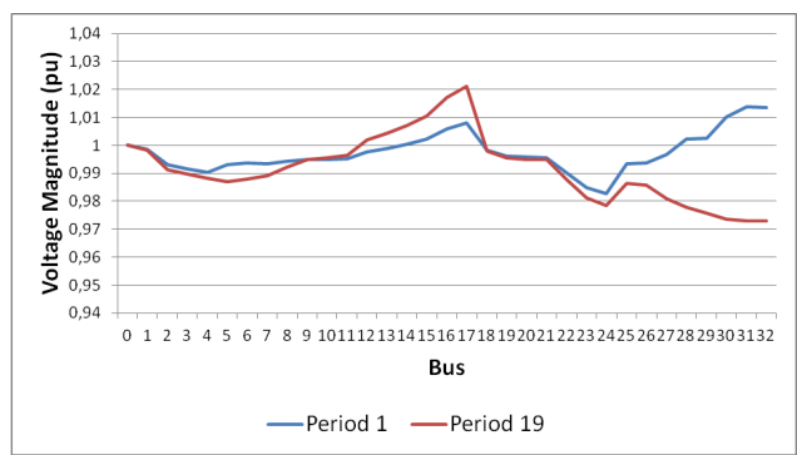

Figure 7. Bus voltage profile in periods 1 and 19 , in scenario 4

Analyzing Figures 3 to 7 it is possible to see the substantial differences between the results of each scenario. The scenario with less power losses is the scenario without reactive power control considering the injection of total reactive power available in capacitor bank of distributed generation. However, these values are result of high quantities of load curtailment. The load curtailment exists in this scenario because the fixed reactive power limits the load flow between some buses. The first scenario has high power losses and the bus voltage magnitude achieves the minimum value permitted ( $0.95 \mathrm{pu})$. In bus 17 and 32 a load curtailment is verified due to the voltage magnitude not being less than the lower voltage magnitude limit. In the scenarios with reactive power control all demand is satisfied. Furthermore, the bus voltage magnitude is acceptable in all bus. When is used the bus voltage variance objective function the power losses are lower. However, the operation cost is lower when the minimizing cost objective function is used.

\section{CONCLUSIONS}

The control of reactive power in distribution network is an important task in future power systems with high penetration of distributed energy resources. An adequate reactive power management allows the increase of more distributed resources amounts and more stability in operation process. Another advantage is the reduction of power losses in the distribution network. These points are shown in the presented case study.

\section{Acknowledgments}

This work is supported by FEDER Funds through COMPETE program and by National Funds through FCT under the projects FCOMP-01-0124-FEDER: PEst-OE/EEI/UI0760/2011, PTDC/EEAEEL/099832/2008, and PTDC/SENENR/099844/2008.

\section{REFERENCES}

[1] S. Khushalani, J. Solanki, and N. Schulz, 2007, "Development of three-phase unbalanced power flow using PV and PQ models for distributed generation and study of the impact of DG models", IEEE Transactions on Power Systems, vol. 22, pp. 1019-1025, 2007.

[2] M.A. Fotouhi Ghazvini, H. Morais, Z. Vale, 2011, "coordination between mid-term maintenance outage decisions and short-term security-constrained scheduling in smart distribution systems", Applied Energy. doi:10.1016/j.apenergy.2011.11.015 (in press).

[3] J. Grainger and W. Stevenson, 1994, "Power system analysis", McGraw-Hill.

[4] Z. Vale, H. Morais, M. Silva, et al., 2009, "Towards a future SCADA", IEEE Power \& Energy Society General Meeting, vol. 1-8, pp. 1894-1900.

[5] Z. Vale, H. Morais, P. Faria, et al., 2010, "Distributed energy resources management with cyber-physical SCADA in the context of future smart grids", Melecon - 2010 15th IEEE Mediterranean Electrotechnical Conference,

[6] H. Morais, P. Kádár, P. Faria, et al., 2010, "Optimal scheduling of a renewable micro-grid in an isolated load area using mixed-integer linear programming", Renewable Energy, vol. 35, pp. 151-156.

[7] T. Sousa, H. Morais, Z. Vale, et al., 2012, "Intelligent energy resource management considering vehicle-to-grid: A simulated annealing approach", Accepted for Publication on IEEE Transaction on Smart Grid, Special Issue on Transportation Electrification and Vehicle-to-Grid Applications, doi: 10.1109/TSG.2011.2165303.

[8] MathWorks, "MATLAB - The language of technical computing", Consulted: January 2012, Available: http://www.mathworks.com/products/matlab/.

[9] GAMS, "GAMS home page", Consulted: January 2012, Available: http://www.gams.com/.

[10] P. Faria, Z. Vale, and J. Ferreira, 2010, "DemSi - A demand response simulator in the context of intensive use of distributed generation", IEEE International Conference on Systems Man and Cybernetics (SMC), pp. 2025-2032. 